Nature of Forcign Bodies removed from the Mcatus.-Beads, peas, stones of truit, pebbles, shells, and pieces of slate pencil are some of the most common substances met with in practice. Amongst the occasional bodies lodged in the auouitory canal we must include larva and mature insects. Small fish have also entered the meatus during bathing. ${ }^{1}$ I have seen several cases in which insects have been unex. pectedly dislodged from the external meatus. Some years since a young lady was brought to me by her father, suffering intense distress from the fluttering of a tiny moth which had become fixed in the cerumen, close to the membrana tympani.

Bodies sometimes impacted for many ycars.-There are casts in record in which foreign substances have been re. taint in the ear for many years. Professor Darling of New York mentions a case in which a pea was lodged in the ear for thirty years. Two years ago I removed a cherrystone from the ear of a woman, which had been allowed to remain there for seventeen years. It was impacted in the meatus, near the membrana tympani, but not actually in contact with it. The hearing power was somewhat impaired.

Extraction of Foreign Bodies. - The vast majority of these accidents may be brought to a successful issue by the simple use of the syringe and warm water. Fortunately other methods are seldom necessary. The treatment should be carried out by a trained nurse, and repeated several times during the day. When difficulty occurs in young children, the surgeon ought to make an effort himself to remove the substance under an anæesthetic. An assistant should hold the head well to the side, and at the same time draw the auricle upwards and backwards, so as to straighten out the canal. In this way the water gets behind the foreign body, and it is soon ejected in the stream.

The Agglutinative Method.-I have never tried this treatment myself, but it has often been practised with success. It consists in fixing a brush or piece of wood to the im. pacted body by means of glue or cobbler's wax, and retaining it in position until firm adhesion has taken place. The substance can then be extracted very easily by careful traction.

Value of Instruments. - Except under special circumstances, aural surgeons are unanimous in condemning the hasty recourse to instrumental assistance. Mr. George Field says instruments are dangerous weapons, and are seldom necessary. In some cases, operations undertaken to extract foreign bodies from the external meatus have been followed by distressing results. Pieces of glass and metal have been pushed down to the bottom of the canal and then through the membrane into the tympanum and Eustachian tube. It must, however, be admitted that cases do occur in which the sub. stance is so firmly impacted in the meatus that extraction is impossible even by the most diligent and skilful application of the syringe. The question then arises, Shall we allow the foreign body to remain in the ear, or make a judicious effort to remove it? The decision must in every case be a matter for earnest consideration. If the substance is very firmly lodged in the external meatus, and is causing pain and tenderness accompanied with chronic inflammatory swelling and discharge, I always decide to operate at once. On several occasions my efforts have been hastened by the anxiety of parents and the fretfulness of the children produced by the persistent use of the syringe. Cases may sometimes fall into our hands in which acute inflammatory changes and tumefaction of the canal have been excited by rough and unsuccessful instrumental treatment. Under these circumstances it is certainly right to defer any interference until these symptoms have been reduced by fomentations and deodorising and soothing remedies.

Instruments for the Removal of Foreign Bodies. - All kinds of tools have been eutployed to assist extraction, including single hooks, screw hooks, with little prongs attached to them, bent probes, pins, wire loops, drills, and forceps of many shapes. I regard the vast majority of such instruments as dangerous weapons. Occasionally a small body, such as a pea or peppercorn, may be gently lifted up by a delicate little hook from the bottom of the meatus, and then ejected from the passage by a stream of warm water.

New Aural Snare - The instrument which I have used with great success in several very urgent cases is represented in the engraving. It consists of two very delicate loops of wire fixed side by side in a metal stem, and these are so fine that when introduced along the meatal wall they

1 St. Roosa, Diseases of the Ear, p. 182. readily slip over the surface of a foreign body. The loops are made in various sizes, and admit of alteration in shape, so that they can be adapted to the varying capacity of the

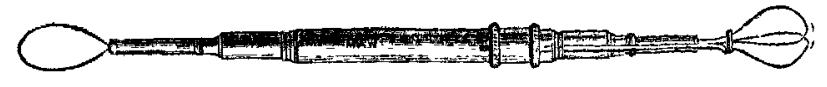

external ear. The snare is especially useful for the extrac tion of hard and round substances, such as small stones, beads, and peas.

Method of performing the Operation.-It is my plan first of all to examine the organ in a good light, and settle the question as to the presence of a foreign body in the canal. 1 then request the parents, if possible, to procure a corresponding body for my examination. The little patient is then placed under the influence of an anæsthetic, and the auricle is firmly drawn upwards and back wards by an assistant. The loops are now introduced and placed in contact with the substance, and then by gentle rotation and pressure they can be readily made to slip over its surface. The sliding collar is now projected to secure it, and extraction carefully practised in the right direction. In cases of severe impaction the loops may some. times slip, so that they have to be reapplied, and another effort performed with greater care and caution. During the extraction smart hamorrhage may take place from the meatal wall, but this is of no importance, for it even facilitates the removal of the substance by reducing the tumefaction of the passage. In cases in which the foreign body is of irregular shape I have found the fine single wire loop, which is attached to the other end of the instrument, of very considerable assistance. The extraction of any substance is always much assisted by filling the. meatus with warm oil, and when the offending substance is. lcdged in the cartilaginous portion of the canal, its ejection can often be promoted by pressure with the fingers around the tragus and gentle manipulation of the auricle. The snare has been very neatly manufactured for me by Messrs. Maw, Son, and Thompson, of London. I have lately usec it successfully in several cases for the removal of foreign bodies from the nasal passages.

Southsea.

\section{A CASE OF}

CARCINOMA OF THE BRAIN, SECONDARY TO THAT OF THE BREAST.

\section{BY CECIL F. BEADLES, M.R.C.S., L.R.C.P.,} HOUSE-SLRGLON TO THE CANCER HOSPITAL.

For permission to publish the notes of this case I am indebted to the kindness of Dr. Snow, under whose care the patient has been during the last five years.

E. D__, a female aged forty.one, widow with two children, with no history of cancer in the family, was. admitted into the Cancer Hospital on Jan. 16th, 1891, with the following past history. On June 11th, 1887, the right breast was amputated for scirrhus, the tumour being the size of a hen's egg, situated in the upper part of the breast: it was hard, freely movable, and not adherent to the skin, and was then of two months' duration. No glands could be felt in the axilla ; it was, however, cleared at the operation, and there were found "no glands appreciably enlarged." On May 1st, 1888, a recurrent nodule the size of a walnut was removed, which was situated along the muscular tissue at the edge of the scapula. The axillary vein was wounded. $0 \mathrm{n}$. Oct. $30 \mathrm{th}, 1888$, a scirrhous nodule the size of a walnut, with a large portion of adherent pectoral muscle, was excised. On Aug. 23rd, 1890, two small glands were removed from the root of the neck, at some distance above the clavicle. They were freely movable, and had been noticed about two weeks; microscopically they were shown to contain carcinoma. The wound did not drain well, and was some time in healing. The patient was discharged on Oct. 6 th, at which time she had occasional slight headache, which was not sufficient to call for treatment.

Present Condition.-Patient has suffered from headache, more or less, from the time she last left the hospital ; it occurs at frequent intervals, and has been far worseduring the last eight weeks. She now has severe headache, coming on at 
night; it is absent during the daytime, and is referred to the vertex and frontal regions. She has no vomiting, but complains of weak eyes. The patient is very dull and forgetful, and sits about without speaking, showing a great contrast to the bright character when last in the hospital, but she is capable of giving a good account of herself. There is a hard enlarged gland felt above the right clavicle, below the seat of the old operation. This she has noticed about three weeks; beyond this there appears no return of the growth. Heart and lungs apparently healthy. Urine: sp. gr. 1012; no albumen or sugar present.-Jan. $22 \mathrm{nd}$ : In the evening the patient had an attack of vomiting, and went to bed. -25 th : The patient still complaining of great headache and inability to sleep at night. She is put on a water-bed. An attempt was made to examine her eyes, but this was found exceedingly difficult. She had to be held up in bed, and her attention could not be directed to any particular object, but there appeared to be slight optic neuritis on the right side.--Feb. 4th : The patient is in a very drowsy condition. She sleeps both day and night, and takes little notice of anything or anybody. There is general weakness, most marked in the legs, but not complete loss of power. There is incontinence of urine and fæces. - March 6th: Much difficulty found in administering food, very little of which is taken. A bedsore has appeared over the sacrum, notwithstanding great care to prevent such. There is paresis of the right side of the body. Tongue furred.-10th : The left pupil is elightly larger than the right. The stupor is less and the tongue less coated.-19th: The left pupil is decidedly larger than the right. There is some conjunctivitis of the right eye.-April 3rd: Both pupils are equal and contracted. Continuous sleep. There is now general paralysis, with rigidity of arms. The paralysis has been very gradual in its onset. The skin presents a rough, dry, stretched appearance. 8th: Patient seems brighter co day and has taken her food better. There is ptosis of left eyelid ; both pupils equal. 13th: Patient has been attempting to talk but did not succeed in making herself understood - 14th: Patient, in reply to questions, can say "yes" or "no." During the last six weeks she has not uttered a word, she has not asked for anybody or taken interest in anything. She can now drink out of the feeder when it is held to her lips.16th: Patient looks much brighter, talks a little; pupils equal, no ptosis.-23rd: For the last four or five days patient has been brighter and more intelligent looking, but she never attempted to speak unless spoken to, and then only replied with "yes" and "no"; paralysis remained complete. She has now relapsed into a drowsy, unconscious state again.25th: Patient is very much worse, groaning expiration, rhonchi heard on both sides of the chest. Right pupil very much contracted, left dilated; neither reacts to light. Pulse 96, irregular. She died later in the day, never baving been out of bed since $J$ an. 22ad (the only day on which vomiting occurred), from which time she had to be fed, only taking about one pint of fluid nourishment daily. The incontinence of urine and frecs continued from the first up to the time of death.

Nesropsy.-The body was slightly emaciated. The skin over the whole body was extremely rough. There was a mass of growth situated over the first three ribs on the right side of the chest; it was attached to the chest wall, and was not fixed to the skin ; it extended outwards to the axilla, and infiltrated the tissues in front of the shoulder. There were some enlarged glands above the right clavicle. There were no secondary deposits in the thoracic and abdo. minal viscera, with the exception of the liver, which contained one small nodule, the size of a pea, situated on the upper surface of the right lobe close to its right border. The right shoulder-joint was disorganised, the cartilage both on the humerus and scapula being eroded and destroyed. On removing the calvaria, the membranes were found congested, and there was an excess of cerebro-spinal fluid. The dura mater was adherent to the left occipital lobe of the brain over a surface of about an inch in diameter. The brain was of normal size, and there was no tumour seen externally. Situated in the posterior of the occipital lobe of the left cerebral hemisphere there was a large deposit of carcinoma; it was of a considerably firmer consistence than the normal brain substance, and was somewhat granular on section, being of a greyish-white colour, distince from that of the brain. This mass was the size of a pullet's egg, nearly two inches in diameter antero-posteriorly and the same ver tically, and reached the posterior surface of the brain, where it was adherent to the dura mater. The upper limit of this growth reached a little above the level of the roof of the a teral ventricle. Another carcinomatous deposit, an inch in diameter, and presenting similar characters to the other, was situated in this hemisphere above and anterior to the Sylvian fissure at Broca's convolution. It reached the exterior of the brain, with some thickening of the pia mater over this part, but no adhesion of the dura mater. The brain substance was firm and healthy in appearance, with no softening and no further deposits of new growth. There was no excess of fluid in the lateral ventricles. The microscopical examination of the growth occurring in the brain showed the greater part of the tumour to be made up of a stroma of homogeneous substance, through which fine fibrils pass, and between these, in places, cells were found either singly or massed together in considerable quantity. These cells were either round or irregular, and vary much in size, some being very large. The stroma contained a large number of thin-walled vessels, and blood was extravasated into parts of the growth. At the edge, more especially where the dura mater was adherent, the growth took the form of typical carcinoma, with large irregular

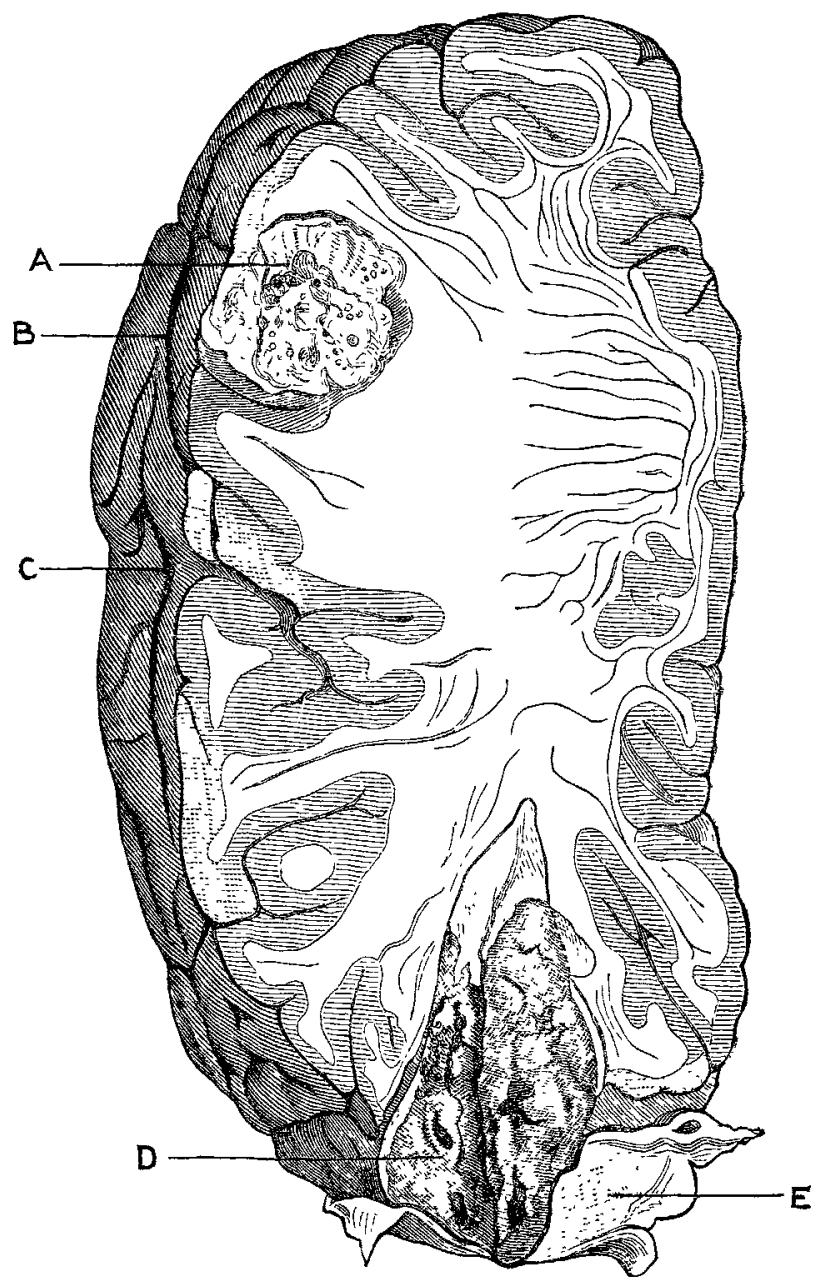

Transverse Section of Left Cerebral Hemisphere, a litTle ABOVE THE LEVEL OF THE ROOF OF LATERAY VENTRICle.

A, Tumour in frontal lobe cut transversely. B, Posterior limb of the Sylvian fissure. $C$, Parallel fissure of temporosphenoidal lobe. $D$, Tumour in occipital lobe, cut vertisphenoidal lobe. D, Tumour in occipital lobe, cut vertilobe. Above is seen the divided superior longitudinal sinus.

cells arranged in alveoli, formed by a coarse fibrous stroma. The alveolar arrangement was only seen to a slight extent in the more internal parts of the tumour, and here the cells were more or less degenerated.

There are several points of interest in this case. It will be seen that the tumour in the occipital lobe occupies what is termed the visual centre, and that the other tumour is situated at what is known as the centre for speech-viz, the posterior extremity of the third frontal convolution and adjacent parts of the ascending frontal of the left cerebral hemisphere. Unfortunately the eyes were never very satisfactorily examined, although several attempts were made; but slight optic neuritis existed on the right sideviz., the opposite to that of the tumour. Dr. Gowers says : "Occasionally it is one-sided, and then it is generally 
on the side opposite the lesion." 1 As to the condition of sight it was almost impossible to learn, owing to the state of stupor in which the patient was in. The stupor and headache were the most, and almost only, symptoms at first. Concerning the former, Dr. (rowers remarks that " when there is pronounced stupor the tumour is usually situated in the cerebral hemisphere, and when there are no other symptoms it is generally in the anterior portion of the frontal lobe." But another symptom existed, which pointed to the anterior of the brain being affected-viz, the loss of speech. This, with the actual discovery after death of a tumour situated at Broca's convolution, together with the temporary improvement, with recovery to some extent of speech shortly before death, form perhaps the most interesting events in the history of the case. " $\Lambda$ peculiar slowness of speech may be met with in association with tumours of almost every position" in the brain; but here we had total loss of speech, lasting from six to eight weeks, pointing to the speech centre being actually involved in the growth. This was followed by a short space of time (nine days) during which there was partial recovery of speech, probably due to the right cerebral hemisphere taking on that function, the patient being over forty years of age. Concerning this, it is worth noting that "loss of speech from disease of the left hemisphere is, as a rule, quickly recovered from in children by the compensatory use of the right hemisphere; a similar recovery takes place in some adults more readilv than in others, and in some it does not take place at all." 2 Vomiting was practically absent, for it only occurred to a very slight extent once, and the patient never had any sense of nausea. No convulsions occurred throughout the course of the disease, and no deafness was noted beyond what might be expected with the stupor. The rarity with which carcinoma occurs, not only in the brain, but throughout the nervous system, secondary to scirrhus of the breast, makes any such case of interest. Out of the large number of persons affected by carcinoma of the breast, few die in hospital, so that it is somewhat difficult to say with what frequency secondaries in the brain do occur; biat as such would usually produce more prominent symptoms than when occurring in other organs, the chances are that if such existed many would fiad their way into hos pitals. As it is, we might expect to find some cases recorded at the Middlesex Hospital owing to their special cancer wards, but for the five years 1882.6 , out of 165 cases of carcinoma of the breast with 44 post mortem examinations, ${ }^{3}$ there was no case with secondary deposit in the brain. Again, at the same hospital, from the post-mortem examinations on 102 consecu tive cases of cancer of the breast for the nine years $1867.75^{4}$ we have only one case of "cancer of brain," although there are four other cases in which there is grow th in cranial bones or the dura mater. Nuan gives $2 l$ post-mortem examinations on breast cases under his own care. In two of these there were tumours in the brain. To only one is there any description: "The brain was, at its base, the seat of a globnlar cancerous tumour, the size of a racquet ball." In this case the patient had deafness, with increasing indis. tinctness of speech and difficulty in walking. Out of 35 cases of cancer of the breast on which post-mortem examinations have been made at the Cancer Hospital during the last three years (May, 1888, to May, 1891), there has only been this single case with growth in the brain. In another case, however, there was a growth springing from the cranial bones, involving the dura mater, and producing a depression on the brain. This, with two somewhat similar specimens, is in the hospital museum. Four years ago a patient died in the Cancer Hospital with scirrhus of the breast, in whom there was found to be a tumour on one of the optic nerves, in size that of the little finger nail, and proving microscopically to be carcinoma; and in 1889 there was a case in which the symptoms pointed to there being a deposit of carcinoma in the spinal cord, secondary to that of the breast, but proved to be a deposit on the bodies of the fifth to the tenth dorsal vertebro. According to Dr. Gowers, true carcinoma is one of the rarer forms of new growth found in the brain, many cases recorded as such belonging in reality to malignant disease of the sarcomatous group. He gives no figures as to its frequency.

1 Diseases of the Nervous System, vol. ii., p. 127. Ibid., vol. ii., p. 103.

3 Roger Williams: Middlesex Hospital Report, 1858 4 Nunn : Cancer of the Breast 1882

\section{TWO CASES OF SCURVY OCCURRING IN CHILDREN.}

BY AMBROSE CHARPENTIER, M.D.

CAses of scurvy are at the present day so rare as to render an account of two sufficiently interesting, I believe, for a place in THE LANCET.

C $\Lambda$ SE 1.-This patient was brought to see me on account of pain and tenderness when moved. The patient was a boy, and soon after his birth nine months ago his mother was unable to suckle him, and gave him cow's milk diluted with water. Then began the sad trouble nsual with artificially fed children. The heavy curds of the cow's milk set up gastric catarrh, so the mother gave condensed milk which, being very dilute, appeared to suit the child better until six months ago (the infant being three months old), when he seemed to crave for more nourish. ment. A "patent" starchy food was administered with the condensed milk, and at all events had the effect of satisfying the child's hunger. On examination the child was rather fat, but flabby and pale, and cried when moved, evidently in pain. The thighs were tender to the touch The fontanelles were quite open, and the ends of the bone were slightly enlarged. The abdomen was swollen. There were no teeth. He was very restless at night, and the head sweated profusely. The bowels acted freely, and the motions were pasty and very offensive. Rickets was diagnosed, and the child was ordered to bave a malted food, with diluted cow's milk in gradually increasing strength, so as to accustom the stomach to better nourishment. Gravy or beef-tea was to be given once a day, and a mixture of iodide of potash and lactophosphate of lime was prescribed to be taken three times a day. A fortnight later he was brought again, showing a small bruise on the back, and later he developed ecchy moses on the eyelids; while the left thigh was swollep hard and brawny, and very tender to the touch. The mouth was very sore, and the gums red and swollen. It was now clear that the case was one of seurvy complicating rickets. A meal of potato pulp twice a day was ordered, as well as raw meat juice and the milk. The improvement was most rapid; in the course of a few days the worst of the sym. ptoms had abated, and a fortnight later he could take iron and cod-liver oil with marked advantage to his health.

C.LSE 2. - This is a case of sporadic cretinism which also developed scurvy. A girl, sixteen years of age, with the tvpical appearance of a cretin, complained of a sore montb. The mother and father are both natives of Middlesex, where they have always lived. The mother knows of nothing in the child's ante-natal life which could account for its con. dition. She states that she was dull at birth, and when seven weeks old had convulsions for a week, and since then has never been like other children. The child is about four feet in height, is dull and apathetic, cannot stand or speak, and takes no interest in life. She is very emaciated, and only swallows liquids. She has a goitre the size of an orange. She has been fed on fresh milk and beef-tea, with minced meat occasionally. I was called to see her on account of her mouth beiog "sore." On examination her gums were swollen, deep reddish-purple in colonr, and prone to bleed. There was a smail braise on the left forearm, one on the left thigh, and a thiid on the right leg, for none of which the mother conld account. There were no other bæmorrhages. The patient was ordered lime-juice to drink and potato pulp three times a day, and a harmaless mixture as a placebo. Two days later the swelling of the gums had disappeared, the bruises were fainter, and no new ones bad come out. In a week's time all the syniptoms of scurvy had disappeared, and the child was in her usual health.

Remarks - The occurrence of these two cases illustrates the necessity of a better knowledge of dietetics in medical practice. It is indeed a subject which well repays any attention given to it, both by the increased power over disease (especially in children) thereby acquired and by the use of more scientific and less haphazard methods of treatment. Another question closely connected with one of these cares is the more common use of condensed milk. In large towas naturally it is more easily obtained than good cow's milk; but even in country places it is largely used, as fresh milk is difficult to obtain. It is undoubtedly very 